\title{
Clinicopathological characteristics and prognostic impact of colorectal cancers with $N R A S$ mutations
}

\author{
TOSHIRO OGURA ${ }^{1,4}$, MIHO KAKUTA ${ }^{2}$, TOSHIMASA YATSUOKA ${ }^{1}$, YOJI NISHIMURA ${ }^{1}$, \\ HIROHIKO SAKAMOTO ${ }^{1}$, KENSEI YAMAGUCHI ${ }^{3}$, MINORU TANABE ${ }^{4}$, \\ YOICHI TANAKA $^{1}$ and KIWAMU AKAGI ${ }^{2}$ \\ Divisions of ${ }^{1}$ Gastroenterological Surgery, ${ }^{2}$ Molecular Diagnosis and Cancer Prevention, and ${ }^{3}$ Gastroenterology, \\ Saitama Cancer Center, Saitama 362-0806; ${ }^{4}$ Department of Hepato-Biliary-Pancreatic Surgery, \\ Graduate School of Medicine, Tokyo Medical and Dental University, Tokyo 113-8519, Japan
}

Received February 17, 2014; Accepted March 22, 2014

DOI: $10.3892 /$ or.2014.3165

\begin{abstract}
At present, molecular markers of colorectal cancer (CRC), including KRAS, NRAS and BRAF mutations, and the microsatellite status are evaluated for the development of personalized treatments. However, clinicopathological and molecular characteristics and the prognostic role of NRAS mutations remain unclear. In the present study, a total of 1,304 consecutive stage 0 -IV CRC tumor samples were analyzed for $K R A S$ (exon 2, 3 and 4), NRAS (exon 2 and 3) and $B R A F$ (exon 15) mutations. Multivariate analysis was performed to assess the prognostic impact of NRAS mutations. KRAS, NRAS and $B R A F$ mutations were identified in 553 (42.4\%), 35 (2.7\%), and $59(4.5 \%)$ of 1,304 CRC cases, respectively. Tumors with $N R A S$ mutations were more frequently located in the distal colorectum compared with those with $K R A S$ or $B R A F$ mutations. Multivariate analysis indicated that $K R A S$ and $B R A F$ mutations were found to be associated with poor prognosis [hazard ratio $(\mathrm{HR})=1.44,95 \%$ confidence interval $(\mathrm{CI}), 1.18$ 1.76 and $\mathrm{HR}=2.09 ; 95 \% \mathrm{CI}, 1.33-3.28$, respectively], whereas NRAS mutations were associated with a trend toward favorable prognosis $(\mathrm{HR}=0.53$; 95\% CI, 0.27-1.03). Characteristics and prognosis of CRC with NRAS mutations are different from those with $K R A S$ or $B R A F$ mutations.
\end{abstract}

\section{Introduction}

The epidermal growth factor receptor (EGFR) is one of the most important molecular targets for advanced colorectal cancer. Activation of this transmembrane receptor tyrosine kinase stimulates signaling pathways supporting cell proliferation, adhesion, migration, evasion of apoptosis, angiogenesis

Correspondence to: Dr Kiwamu Akagi, Division of Molecular Diagnosis and Cancer Prevention, Saitama Cancer Center, 818 Komuro, Ina-machi, Kitaadati-gun, Saitama 362-0806, Japan

E-mail: akagi@cancer-c.pref.saitama.jp

Key words: NRAS, KRAS, BRAF, colorectal cancer, microsatellite instability and survival (1-3). Oncogenic signaling pathways downstream of EGFR, including RAS/Raf/MAPK and PI3K/PTEN/Akt pathways, are important mechanisms of tumor progression.

Activating mutations in the $R A S$ oncogene family are present in $\sim 30 \%$ of all human cancers. RAS genes encode highly homologous proteins: KRAS, NRAS and HRAS (4). Mutations in the KRAS gene are frequency reported in various human neoplasms, including pancreatic cancer, biliary tract cancer and lung adenocarcinoma (4-6). Cancer types with a high rate of NRAS mutations include myeloid leukemia and cutaneous melanoma, whereas $H R A S$ mutations are typical of bladder and cervical cancers $(4,7,8)$. In colorectal cancer (CRC), rates of KRAS, NRAS and HRAS mutation are 30-42\%, $2.2-5 \%$ and $0-0.8 \%$, respectively (9-14). Relatively low rates of $N R A S$ and HRAS mutations in CRC remain unexplained.

Anti-EGFR antibody therapy exhibits antitumor effects by inhibiting multiple EGFR signaling pathways, including RAS/RAF/MAPK and PI3K/PTEN/AKT pathways. Clinical trials have demonstrated that anti-EGFR monoclonal antibodies (i.e., cetuximab or panitumumab) are largely ineffective for metastatic CRC patients when tumors harbor mutations in the codon 12 or 13 of KRAS exon 2 (15-20). These mutations cause constitutive activation of the RAS/RAF/MAPK pathway, regardless of EGFR inhibition. Therefore, KRAS exon 2 mutations are recognized as predictive markers of anti-EGFR therapy resistance for metastatic CRC patients. Accordingly, these clinical trials routinely exclude CRC patients harboring KRAS exon 2 mutations.

Recent studies suggest that other activating mutations in $K R A S$ or $N R A S$, in addition to KRAS exon 2, confer resistance to anti-EGFR therapy $(9,11,13,21)$. Since KRAS and NRAS mutations tend to be mutually exclusive, they may be present in approximately half of metastatic CRC patients (9-14). Therefore, personalized cancer therapy should be tailored to the KRAS and NRAS mutation profile of each patient to improve treatment outcomes.

Previous studies have evaluated the clinicopathological features and prognostic influence of KRAS or BRAF mutations in colorectal cancer. The prognostic value of KRAS mutations in CRC remains controversial (22-24). In contrast, $B R A F$ mutations are associated with proximal colon tumor location, 
poor differentiation, mucinous component and microsatellite instability. Patients with $B R A F$-mutated tumors revealed lower survival rates compared with wild-type tumors, particularly those with $B R A F$-mutated and microsatellite-stable CRC $(22,25-27)$. On the other hand, clinicopathological characteristics, molecular features, and the prognostic value of the NRAS mutation remain largely unknown $(10,12)$. To date, analyses of NRAS mutations in colorectal cancer were performed as part of a subset analysis of clinical studies for treatment of metastatic CRC with anti-EGFR antibodies, and few studies have described NRAS mutations in the early stage of CRC. Irahara et al (12) associated NRAS mutations with left-sided cancers in females, but the data did not reach statistical significance since NRAS mutations were only detected in $5(2.2 \%)$ of the 225 cases. Therefore, the prognostic role and clinical characteristics of NRAS mutations should be clarified using large tissue samples to guide future clinical studies on the predictive impact of the NRAS gene.

The present study used 1,304 consecutive samples of stage 0 -IV CRC to investigate the impact of mutations in NRAS exon 2 and 3 , in addition to $K R A S$ and $B R A F$. We evaluated the relationship between NRAS mutations and other clinicopathological or molecular features, including $K R A S$ and $B R A F$ mutations, microsatellite instability (MSI) status and patient survival.

\section{Materials and methods}

Patients and tissue samples. The present study was conducted on 1,304 consecutive primary CRC patients at the Saitama Cancer Center from July 1999 to July 2008. Information on clinical data, including age at diagnosis, gender, tumor size, histological differentiation, tumor location, International Union against Cancer (UICC) stage and prognosis were collected from medical records. Tissue samples were surgically excised after obtaining informed consent from each patient. All tumor tissues were paired with normal colorectal tissues and immediately stored at $-80^{\circ} \mathrm{C}$. The present study was approved by the Ethics Committee of the Saitama Cancer Center.

Mutation analysis of KRAS, BRAF and NRAS. Genomic DNA from each sample was extracted by standard SDS-proteinase K procedure, followed by ethanol precipitation. All tumor samples were tested for $K R A S$ exon 2, 3 and 4; BRAF exon 15 (codon 600); NRAS exon 2 and 3; and MSI status.

$K R A S$ mutations in exon 2 and 3 were detected by denaturing gradient gel electrophoresis (DGGE), and $B R A F$ mutations in exon 15 by PCR-restriction fragment length polymorphism (RFLP), as previously described $(28,29)$.

High resolution melting (HRM) analysis was used to identify mutations in NRAS exon 2 and 3 and in KRAS exon 4 using a Rotor-Gene Q (Qiagen, Hilden, Germany). Primer sets for NRAS were as follows: exon 2, 5'-GGTTTCCAACAGGT TCTTGC-3' (forward) and 5'-CACTGGGCCTCACCTCTA TG-3' (reverse); exon 3, 5'-CACACCCCCAGGATTCTTAC-3' (forward) and 5'-TGGCAAATACACAGAGGAAGC-3' (reverse). The primer set for KRAS exon 4 was as follows: 5'-GCCTTCTAGAACAGTAGACAC-3' (forward) and 5'-GA CATAACAGTTATGATTTTGCAGA-3' (reverse). The reac- tion mixture contained $7 \mu \mathrm{l}$ of $2 \mathrm{X}$ LightCycler $480 \mathrm{High}$ Resolution Melting Master Reaction Mix (Roche Diagnostics, Mannheim, Germany) with $0.21 \mu \mathrm{M}$ of each forward and reverse primer, $3.2 \mathrm{mM} \mathrm{MgCl}_{2}, 20 \mathrm{ng}$ purified genomic DNA, and water to a total volume of $14 \mu \mathrm{l}$. PCR cycling and melting conditions were as follows: initial denaturation at $95^{\circ} \mathrm{C}$ for $5 \mathrm{~min}$, followed by 40 cycles of $10 \mathrm{sec}$ at $95^{\circ} \mathrm{C}, 20 \mathrm{sec}$ at $57^{\circ} \mathrm{C}$, and $10 \mathrm{sec}$ at $72^{\circ} \mathrm{C}$. One heteroduplex cycle was performed at $95^{\circ} \mathrm{C}$ for $1 \mathrm{~min}$ and $40^{\circ} \mathrm{C}$ for $1 \mathrm{~min}$, followed by melting from $72^{\circ} \mathrm{C}$ to $95^{\circ} \mathrm{C}$ with 10 acquisitions per ${ }^{\circ} \mathrm{C}$. HRM data were analyzed using the Rotor-Gene Q software ver.2.0.2.4.

The DNA sequence of NRAS exon 2 and 3 mutations was determined by HRM using primers particularly designed for HRM. Amplified products were labeled with GenomeLab ${ }^{\mathrm{TM}}$ DTCS Quick Start kit (Beckman Coulter Inc., Fullerton, CA, USA) according to the manufacturer's instructions and sequenced using the GenomeLab ${ }^{\mathrm{TM}} \mathrm{GeXP}$ Genetic Analysis System (Beckman Coulter). Sequencing was performed in both directions, and sequence analysis was performed using the GenomeLab Genetic Analysis System v10.2 (Beckman Coulter).

Analysis of microsatellite status. The MSI status was determined using Bethesda markers: BAT25, BAT26, D5S346, D2S123 and D17S250. PCR and subsequent analyses were performed as previously described (30). CRC samples showing instability in two or more markers were defined as microsatellite instability-high (MSI-H), and the ones with none or one marker as microsatellite stable (MSS).

Statistical analysis. Possible associations between each mutation and clinicopathological parameters of CRC were assessed by the Chi-square or Fisher's exact test for categorical variables and Mann-Whitney U or Kruskal-Wallis test for continuous variables. Overall survival (OS) time was calculated from the date of surgery to the date of death by any cause or censored at the last follow-up visit. Cox proportional hazards analysis was used to estimate clinicopathological- and biomarker-specific survival hazard ratios (HRs) and $95 \%$ confidence intervals (CIs). A multivariable model stratification by UICC stage was performed. All P-values were calculated from two-sided test, and P-values < 0.05 were considered statistically significant. All statistical analyses were performed with the SPSS Statistics v.20 (SPSS, Inc., Chicago, IL, USA).

\section{Results}

Patient characteristics. All 1,304 patients enrolled in the present study were diagnosed with either CRC stage $0(n=48)$, stage I $(n=248)$, stage II $(n=407)$, stage III $(n=384)$ or stage IV $(n=217)$ (Table I). Three hundred and seventy-nine cancers were from the proximal colon (cecum to transverse colon), 544 from the distal colon (descending colon to sigmoid colon) and 381 from the rectum. The median follow-up period was 5.6 years (interquartile range, 4.1-7.8 years), during which there were 435 deaths $(33 \%)$.

Frequency of KRAS, NRAS and BRAF mutations. All 1,304 CRC cases were examined for mutations in KRAS (exon 2, 3 and 4), NRAS (exon 2 and 3 ) and BRAF (exon 15), as well 
Table I. Clinicopathological and molecular features of all of the CRC samples.

\begin{tabular}{|c|c|}
\hline Features & $\begin{array}{c}\text { Patients } \\
(\mathrm{n}=1,304) \\
\mathrm{n}(\%)\end{array}$ \\
\hline \multicolumn{2}{|l|}{ Gender } \\
\hline Male & $780(59.8)$ \\
\hline Female & $524(40.2)$ \\
\hline Age \pm SD (years) & $63.8 \pm 10.4$ \\
\hline \multicolumn{2}{|l|}{ Location } \\
\hline Proximal & $379(29.1)$ \\
\hline Distal & $544(41.7)$ \\
\hline Rectum & $381(29.2)$ \\
\hline \multicolumn{2}{|l|}{ Tumor size } \\
\hline Mean $\pm \mathrm{SD}(\mathrm{mm})$ & $45.4 \pm 24.2$ \\
\hline \multicolumn{2}{|l|}{ Histological features } \\
\hline Well-differentiated & $144(11.0)$ \\
\hline Moderately differentiated & $1,078(82.7)$ \\
\hline Poorly differentiated & $34 \quad(2.6)$ \\
\hline Others & $48 \quad(3.7)$ \\
\hline \multicolumn{2}{|l|}{ Stage } \\
\hline 0 & $48 \quad(3.7)$ \\
\hline 1 & $248(19.0)$ \\
\hline 2 & $407(31.3)$ \\
\hline 3 & $384(29.4)$ \\
\hline 4 & $217(16.6)$ \\
\hline \multicolumn{2}{|l|}{$K R A S$ status } \\
\hline Mutated-type & $553(42.4)$ \\
\hline Wild-type & $751(57.6)$ \\
\hline \multicolumn{2}{|l|}{ NRAS status } \\
\hline Mutated-type & $35 \quad(2.7)$ \\
\hline Wild-type & $1,269(97.3)$ \\
\hline \multicolumn{2}{|l|}{$B R A F$ status } \\
\hline Mutated-type & $59(4.5)$ \\
\hline Wild-type & $1,245(95.5)$ \\
\hline \multicolumn{2}{|l|}{ MSI status } \\
\hline MSI-H & $72 \quad(5.5)$ \\
\hline MSS & $1,232(94.5)$ \\
\hline
\end{tabular}

SD, standard deviation; MSI, microsatellite instability; MSI-H, microsatellite instability-high; MSS, microsatellite stable.

as MSI status and clinicopathological factors (Table I). KRAS mutations were detected in $42.4 \%(\mathrm{n}=553), N R A \mathrm{~S}$ in $2.7 \%$ $(\mathrm{n}=35)$ and $B R A F$ in $4.5 \%(\mathrm{n}=59)$ of patients. MSI-H was detected in $5.5 \%(n=72)$ of cases. Table II presents changes in the nucleotides and corresponding amino acids detected in $N R A S$, with p.G12D (codon 12), p.G13R (codon 13) and p.Q61K (codon 61) as the most frequently noted mutations.

Mapping associations between molecular markers revealed that 3 patients had both KRAS and NRAS muta-
Table II. Frequency and type of NRAS mutation.

\begin{tabular}{|c|c|c|c|}
\hline $\begin{array}{l}\text { Nucleotide } \\
\text { mutation }\end{array}$ & $\begin{array}{l}\text { Amino } \\
\text { acid change }\end{array}$ & $\begin{array}{c}\text { Mutation } \\
\text { frequency (n) }\end{array}$ & $\begin{array}{l}\text { Coincidental } \\
K R A S \text { mutation }\end{array}$ \\
\hline \multicolumn{4}{|l|}{ Codon12 } \\
\hline c. $35 \mathrm{G}>\mathrm{A}$ & p.G12D & $20.0 \% \quad(7)$ & \\
\hline c. $34 \mathrm{G}>\mathrm{T}$ & p.G12C & $5.7 \%$ & \\
\hline c. $35 \mathrm{G}>\mathrm{T}$ & p.G12V & $5.7 \% \quad(2)$ & \\
\hline \multicolumn{4}{|l|}{ Codon13 } \\
\hline c. $37 \mathrm{G}>\mathrm{C}$ & p.G13R & $11.0 \% \quad$ (4) & \\
\hline c. $38 \mathrm{G}>\mathrm{A}$ & p.G13D & $8.6 \% \quad(3)$ & p.G57T \\
\hline c. $38 \mathrm{G}>\mathrm{T}$ & p.G13V & $2.9 \% \quad(1)$ & \\
\hline \multicolumn{4}{|l|}{ Codon61 } \\
\hline c. $181 \mathrm{C}>\mathrm{A}$ & p.Q61K & $26 \% \quad(9)$ & \\
\hline c. $182 \mathrm{~A}>\mathrm{T}$ & p.Q61L & $5.7 \% \quad(2)$ & \\
\hline c. $183 \mathrm{~A}>\mathrm{C}$ & p.Q61H & $2.9 \% \quad(1)$ & \\
\hline c. $183 \mathrm{~A}>\mathrm{T}$ & p.Q61H & $2.9 \% \quad(1)$ & \\
\hline c. $182 \mathrm{~A}>\mathrm{G}$ & p.Q61R & $2.9 \% \quad(1)$ & \\
\hline \multicolumn{4}{|l|}{ Codon9 } \\
\hline c. $26 \mathrm{~T}>\mathrm{C}$ & p.V9A & $2.9 \% \quad(1)$ & p.G12D \\
\hline \multicolumn{4}{|l|}{ Codon68 } \\
\hline c. $204 \mathrm{~A}>\mathrm{T}$ & p.R68S & $2.9 \% \quad(1)$ & p.G12V \\
\hline
\end{tabular}

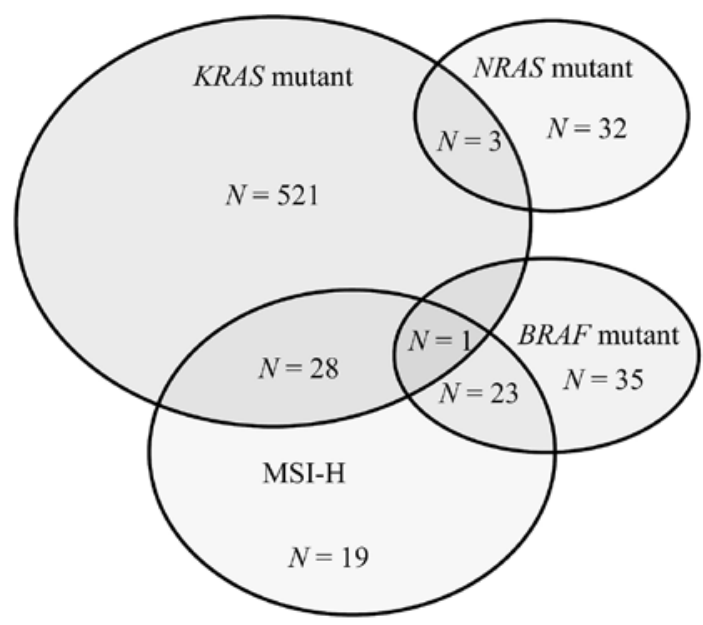

Figure 1. Associations between KRAS, NRAS and BRAF mutations and MSI. MSI-H, microsatellite instability-high.

tions, whereas 1 patient had both KRAS and $B R A F$ mutations (Fig. 1). KRAS/NRAS mutation combinations were as follows: p.G12D/p.V9A, pG12V/p.R68S and p.G57T/p.G13D. In contrast, NRAS and BRAF mutations were mutually exclusive. Regarding the MRI status, 28 patients with KRAS mutations also had MSI-H tumors compared with 23 patients with $B R A F$ mutations. None of the patients with NRAS mutations had MSI-H tumors.

$B R A F$ mutations were significantly more frequent in MSI-H than in MSS tumors $(\mathrm{P}<0.001)$, whereas no significant association was observed between MSI status and KRAS or NRAS mutations. 
Table III. Mutation rates of $K R A S$ and $N R A S$ genes for each exon.

\begin{tabular}{|c|c|}
\hline Gene & Patients with mutations, $\mathrm{n}(\%)$ \\
\hline \multicolumn{2}{|l|}{$K R A S$} \\
\hline Exon 2 & $495(38.0)$ \\
\hline Exon 3 & $26(2.0)$ \\
\hline Exon 4 & $32(2.5)$ \\
\hline \multicolumn{2}{|l|}{ NRAS } \\
\hline Exon 2 & $20 \quad(1.5)$ \\
\hline Exon 3 & $15(1.2)$ \\
\hline
\end{tabular}

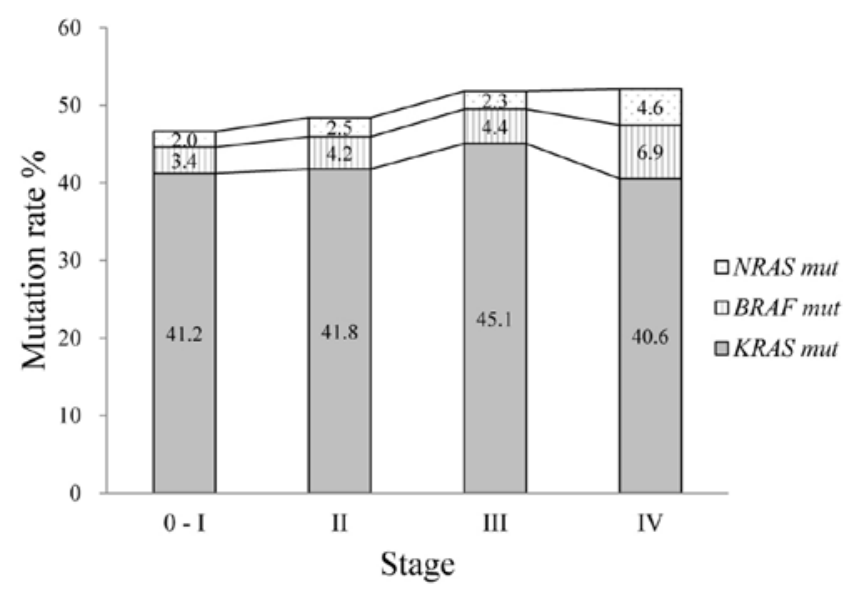

Figure 2. Mutation rates of the KRAS, NRAS and BRAF genes according to UICC staging.

Frequency of KRAS and NRAS mutations in each exon. In the KRAS gene, most mutations were located in exon 2, with 495 of 1,304 cases $(38.0 \%)$, whereas exon 3 or 4 mutations were detected in $26(2.0 \%)$ and $32(2.5 \%)$ cases, respectively (Table III). In the NRAS gene, 20 (1.5\%) mutations were identified in exon 2 and $15(1.2 \%)$ mutations in exon 3.

Impact of KRAS, NRAS and BRAF mutation status on clinicopathological and molecular characteristics of the colorectal cancer patients. CRC patients were categorized into three groups on the basis of KRAS, NRAS and BRAF mutations, and they were compared in terms of gender, age, colorectal tumor location, tumor maximum size, histological differentiation, mucinous component, depth of tumor invasion, UICC stage, extramural venous invasion and MSI status (Table IV). $B R A F$-mutated tumors were more frequently associated with mucinous component tumors (KRAS, $\mathrm{P}=0.003$; NRAS, $\mathrm{P}=0.002)$, poorly differentiated tumors $(K R A S, \mathrm{P}<0.001$; $N R A S, \mathrm{P}=0.013)$, female gender $(K R A S, \mathrm{P}=0.022)$ and MSI-H (KRAS and NRAS, $\mathrm{P}<0.001) . N R A S$-mutated tumors were more frequently located in the distal colorectum compared with $K R A S$ - or $B R A F$-mutated tumors $(\mathrm{P}=0.015$ and $\mathrm{P}<0.001$, respectively). Compared with triple wild-type tumors (KRAS, $N R A S$ and $B R A F$ wild-type), $K R A S$ - and $B R A F$-mutated tumors were more commonly noted in the proximal colon
( $\mathrm{P}<0.001$ and $\mathrm{P}<0.001$, respectively), whereas no significant difference was observed between NRAS-mutated tumors and triple wild-type tumors $(\mathrm{P}=0.201)$.

Mutation rates of KRAS, NRAS and BRAF for each UICC stage are presented in Fig. 2. KRAS mutations were detected at similar frequencies in stage 0 -I to IV. NRAS mutations tended to occur more frequently in stage IV cancers than in stage 0 -III cancers compared with KRAS mutations $(\mathrm{P}=0.061)$.

Impact of KRAS, NRAS and BRAF mutations on CRC patient survival. Univariate analysis was conducted in regards to age, gender, tumor location, stage, histological subtype, mucinous component, extramural venous invasion, MSI status, $K R A S$, $N R A S$ and $B R A F$ mutations (Table V). Patients with KRAS and $B R A F$ mutations had significantly worse survival compared with wild-type cases $[\mathrm{HR}=1.25 ; 95 \%$ confidence interval (CI) 1.03-1.52; $\mathrm{P}=0.027$ and $\mathrm{HR}=1.73 ; 95 \% \mathrm{CI}, 1.15-2.60 ; \mathrm{P}=0.009$, respectively]. Four other variables were significantly associated with poor prognosis, namely age $\geq 65$ years ( $\mathrm{HR}=1.39$; 95\% CI, 1.15-1.69; P=0.001), UICC stage (stage II: HR=2.33; stage III: HR=3.58; stage IV: HR=14.14; $\mathrm{P}<0.001$ respectively), histological subtype (HR=1.82; 95\% CI, 1.31-2.52; $\mathrm{P}<0.001$ ), and extramural venous invasion $(\mathrm{HR}=3.28 ; 95 \% \mathrm{CI}, 2.50-4.30$; $\mathrm{P}<0.001)$. The only predictor of good prognosis was female gender ( $\mathrm{HR}=0.73$; 95\% CI, 0.60-0.89; $\mathrm{P}=0.002$ ). In multivariable analysis, $K R A S$ and $B R A F$ mutations were associated with significantly higher mortality rates when stratified according to UICC staging ( $\mathrm{HR}=1.44 ; 95 \% \mathrm{CI}, 1.18-1.79 ; \mathrm{P}<0.001$ and $\mathrm{HR}=2.09 ; 95 \%$ CI, 1.36-3.28; $\mathrm{P}=0.001$, respectively). Notably, NRAS-mutated tumors demonstrated a trend towards favorable prognosis $(\mathrm{HR}=0.53 ; 95 \% \mathrm{CI}, 0.27-1.03 ; \mathrm{P}=0.059)$.

\section{Discussion}

The present study investigated clinicopathological and prognostic features of $K R A S, N R A S$ and $B R A F$ mutations in tumors from 1,304 consecutive CRC patients. An important finding was that patients undergoing CRC tumor resection at all stages were targeted by all these mutations. In addition, to the best of our knowledge, this is the first large study to present statistically significant comparisons between these three categories of $R A S / R A F$ mutations in CRC patients.

NRAS mutations were observed in 35 (2.7\%) of the 1,304 patients, of which $20(1.5 \%)$ patients revealed a mutation in exon 2 and others (1.2\%) in exon 3 . These data are consistent with previous studies that reported NRAS mutations in 2.2-5.0\% of CRC tumors, with approximately equal frequency in exon 2 and $3(9,10,12,29)$. Moreover, we showed that NRAS mutations are detected in early stages of CRC and tend to occur more frequently in stage IV cancers than in stage 0-III cancers. Therefore, NRAS mutations appear to be acquired at early and advanced stages of CRC (31). Nonetheless, the tendency of higher NRAS mutation rates in stage IV CRC should be ascertained in a larger scale study. The frequency of KRAS mutations was also compatible with previous studies, but the frequency of $B R A F$ mutations (4.5\%) declined below $7.4-10.1 \%$ of the values previously reported $(10,13,14)$. On the other hand, Yokota et al (32) reported an incidence of $4.7 \%$ for $B R A F$ mutations (15/319 patients) in Japanese CRC patients. Such agreement with our Japanese study suggests that racial 
Table IV. Clinicopathological characteristics according to the KRAS, NRAS and BRAF mutation status.

\begin{tabular}{|c|c|c|c|c|c|c|c|}
\hline \multirow[b]{2}{*}{ Characteristics } & \multirow{2}{*}{$\begin{array}{c}\text { Triple } \\
\text { wild-type } \\
\text { n }(\%)\end{array}$} & \multirow[b]{2}{*}{$\begin{array}{c}\text { KRAS mt } \\
\mathrm{n}(\%)\end{array}$} & \multirow[b]{2}{*}{$\begin{array}{c}N R A S \mathrm{mt} \\
\mathrm{n}(\%)\end{array}$} & \multirow[b]{2}{*}{$\begin{array}{c}B R A F \mathrm{mt} \\
\mathrm{n}(\%)\end{array}$} & \multicolumn{3}{|c|}{ P-value } \\
\hline & & & & & $\begin{array}{c}K R A S \text { vs. } \\
N R A S\end{array}$ & $\begin{array}{c}K R A S \text { vs. } \\
B R A F\end{array}$ & $\begin{array}{c}N R A S \text { vs. } \\
B R A F\end{array}$ \\
\hline Patient & 661 & & & & 0.222 & 0.022 & 0.633 \\
\hline Male & $431(65.2)$ & $309(55.9)$ & $16(45.7)$ & $24(40.7)$ & & & \\
\hline Female & $230(34.8)$ & $244(44.1)$ & $19(54.3)$ & $35(59.3)$ & & & \\
\hline Age $\pm \mathrm{SD}$ (years) & $63.3 \pm 10.3$ & $64.2 \pm 10.4$ & $65.5 \pm 9.5$ & $64.2 \pm 11.5$ & 0.716 & 0.64 & 0.997 \\
\hline Location & & & & & 0.015 & $<0.001$ & $<0.001$ \\
\hline Proximal & $142(21.4)$ & $189(34.2)$ & $4(11.4)$ & $46(77.9)$ & & & \\
\hline Distal & $311(47.1)$ & $209(37.8)$ & $16(45.7)$ & $9(15.3)$ & & & \\
\hline Rectum & $208(31.5)$ & $155(28.0)$ & $15(42.9)$ & $4(6.8)$ & & & \\
\hline Tumor size & & & & & 0.456 & 0.417 & 0.928 \\
\hline Mean \pm SD $(m m)$ & $44.1 \pm 24.3$ & $46.1 \pm 22.7$ & $48.0 \pm 23.0$ & $52.6 \pm 33.9$ & & & \\
\hline Histologic feature & & & & & 0.916 & $<0.001$ & 0.013 \\
\hline Well-differentiated & $58(8.8)$ & $78(14.1)$ & $6(17.1)$ & $3(5.1)$ & & & \\
\hline Moderately differentiated & $573(86.6)$ & $437(79.0)$ & $29(82.9)$ & $41(69.4)$ & & & \\
\hline Poorly differentiated & $16(2.4)$ & $11(2.0)$ & $0 \quad(0.0)$ & $8(13.6)$ & & & \\
\hline Mucinous & $11 \quad(1.7)$ & $25(4.5)$ & $0 \quad(0.0)$ & $7(11.9)$ & & & \\
\hline Others & $3(0.5)$ & $2(0.4)$ & $\begin{array}{ll}0 & (0.0)\end{array}$ & $0 \quad(0.0)$ & & & \\
\hline Mucinous component & & & & & 0.068 & 0.003 & 0.002 \\
\hline+ & $34 \quad(5.1)$ & $98(17.7)$ & $2(5.7)$ & $20(33.9)$ & & & \\
\hline- & $627(94.9)$ & $455(82.3)$ & $33(94.3)$ & $39(66.1)$ & & & \\
\hline Depth of tumor invasion & & & & & 0.63 & 0.483 & 0.838 \\
\hline Tis & $15 \quad(2.3)$ & $33(6.0)$ & $\begin{array}{ll}0 & (0.0)\end{array}$ & $\begin{array}{ll}0 & (0.0)\end{array}$ & & & \\
\hline $\mathrm{T} 1$ & $62(9.4)$ & $45 \quad(8.1)$ & $4(11.4)$ & $4(6.8)$ & & & \\
\hline $\mathrm{T} 2$ & $110(16.6)$ & $68(12.3)$ & $3(8.6)$ & $7(11.9)$ & & & \\
\hline $\mathrm{T} 3$ & $404(61.1)$ & $352(63.7)$ & $24(68.6)$ & $39(66.0)$ & & & \\
\hline $\mathrm{T} 4$ & $70(10.6)$ & $55 \quad(9.9)$ & $4(11.4)$ & $9(15.3)$ & & & \\
\hline UICC stage & & & & & 0.353 & 0.151 & 0.75 \\
\hline 0 & $15(2.3)$ & $33(6.0)$ & $\begin{array}{ll}0 & (0.0)\end{array}$ & $0 \quad(0.0)$ & & & \\
\hline 1 & $143(21.6)$ & $89(16.1)$ & $6(17.1)$ & $10(16.9)$ & & & \\
\hline 2 & $210(31.8)$ & $170(30.7)$ & $10(28.6)$ & $17(28.8)$ & & & \\
\hline 3 & $188(28.4)$ & $173(31.3)$ & $9(25.7)$ & $17(28.8)$ & & & \\
\hline 4 & $105(15.9)$ & $88(15.9)$ & $10(28.6)$ & $15(25.5)$ & & & \\
\hline Extramural venous invasion & & & & & 0.231 & 0.105 & 0.689 \\
\hline+ & $471(71.3)$ & $365(66.0)$ & $24(68.6)$ & $46(78.0)$ & & & \\
\hline- & $190(28.7)$ & $188(34.0)$ & $11(31.4)$ & $13(22.0)$ & & & \\
\hline MSI-H & & & & & 0.171 & $<0.001$ & $<0.001$ \\
\hline+ & $20 \quad(3.0)$ & $29(5.2)$ & $0(0.0)$ & $24(40.7)$ & & & \\
\hline- & $641(97.0)$ & $524(94.8)$ & $35(100)$ & $35(59.3)$ & & & \\
\hline
\end{tabular}

MSI-H, microsatellite instability-high.

or environmental factors may affect the frequency of $B R A F$ mutations. The strong overlap between $B R A F$ mutation and MSI-H status that we detected suggests that the low frequency in the $B R A F$ mutation is affected by the low MSI-H frequency reported among Asians compared with Westerners (33).

In the present study, 3 cases of 1,304 had mutations in both $K R A S$ and NRAS, which is inconsistent with previous reports of mutual exclusivity. Notably, all three tumors presented rare mutations (NRAS p.V9A, NRAS p.R68S and KRAS p.G57T), whereas these tumors had common mutations (KRAS p.G12D, $K R A S$ p.G12V and NRAS p.G13D). The oncogenic activity of these minor mutations is unknown, except for KRAS p.G57T (28). Therefore, KRAS and NRAS mutation detection only in major mutation lesion may have missed these rare mutations. 
Table V. Univariate and multivariate analyses of the covariates associated with overall survival.

\begin{tabular}{|c|c|c|c|c|}
\hline Covariates & $\begin{array}{l}\text { Univariate } \\
\mathrm{HR}(95 \% \mathrm{CI})\end{array}$ & $\mathrm{P}$-value & $\begin{array}{l}\text { Multivariate } \\
\text { HR(95\% CI })\end{array}$ & P-value \\
\hline Age $\geq 65$ years & $1.39(1.15-1.69)$ & 0.001 & $1.53(1.27-1.86)$ & $<0.001$ \\
\hline Female & $0.73(0.60-0.89)$ & 0.002 & $0.69(0.56-0.84)$ & $<0.001$ \\
\hline $\begin{array}{l}\text { Tumor location } \\
\text { (proximal vs. distal colorectum) }\end{array}$ & $0.88(0.72-1.08)$ & 0.22 & $1.01(0.81-1.25)$ & 0.96 \\
\hline$K R A S$-mutant & $1.25(1.03-1.52)$ & 0.027 & $1.44(1.18-1.76)$ & $<0.001$ \\
\hline$N R A S$-mutant & $0.83(0.43-1.61)$ & 0.57 & $0.53(0.27-1.03)$ & 0.059 \\
\hline$B R A F$-mutant & $1.73(1.15-2.60)$ & 0.009 & $2.09(1.33-3.28)$ & 0.001 \\
\hline $\begin{array}{l}\text { MSS } \\
\text { (vs. MSI-high) }\end{array}$ & $1.59(0.98-2.58)$ & 0.062 & $1.56(0.92-2.64)$ & 0.10 \\
\hline \multicolumn{5}{|l|}{ UICC stage } \\
\hline II & $2.33(1.58-3.44)$ & $<0.001$ & $-{ }^{\mathrm{a}}$ & \\
\hline III & $3.58(2.46-5.22)$ & $<0.001$ & $-{ }^{\mathrm{a}}$ & \\
\hline $\begin{array}{l}\text { IV } \\
\text { (vs. Stage } 0 \text { and I) }\end{array}$ & $14.14(9.68-20.7)$ & $<0.001$ & $-{ }^{\mathrm{a}}$ & \\
\hline $\begin{array}{l}\text { Histological subtype } \\
\text { (vs. well and mod ) }\end{array}$ & $1.82(1.31-2.52)$ & $<0.001$ & $1.59(1.09-2.32)$ & 0.016 \\
\hline Mucinous component & $1.23(0.93-1.62)$ & 0.14 & $0.81(0.59-1.11)$ & 0.2 \\
\hline Extramural venous invasion & $3.28(2.50-4.30)$ & $<0.001$ & $1.78(1.31-2.37)$ & $<0.001$ \\
\hline
\end{tabular}

${ }^{a}$ UICC Stage was a stratifying variable in the multivariate analysis. HR, hazard ratio.

Colorectal tumors with NRAS mutations were found more frequently in the distal colon and rectum compared with tumors with KRAS or BRAF mutations, while the distribution of $B R A F$-mutated tumors was consistent with previous studies reporting that $B R A F$-mutated tumors are primarily located in the proximal colon $(22,32,34)$. It was proposed that cellular transformation and mutations occur more frequently in the proximal colon due to close contact of epithelial cells with stimulating bowel content (34). However, this theory does not explain the higher frequency of NRAS-mutated tumors in the distal colon and rectum compared with those with KRAS or $B R A F$ mutations. Elucidating factors responsible for distinct locations of $K R A S$ - and NRAS-mutated tumors may be crucial to our understanding of NRAS mutations in CRC patients.

The prognosis of advanced CRC patients carrying NRAS mutations has been reported in the form of subset analysis of clinical studies using anti-EGFR drugs such as cetuximab and panitumumab $(9,13,14)$. Although there is currently no consistent view regarding the efficacy of anti-EGFR drugs for CRC patients with NRAS mutations, several reports consider this form of therapy inappropriate for such CRC patients. However, the fundamental malignant potential of the NRAS mutation should be considered to determine the efficacy of anti-EGFR drugs. To the best of our knowledge, this is the first study to compare patient survival in $N R A S$-mutated and triple wild-type CRCs. Numerous studies report an association between $B R A F$ mutations and poor clinical outcome $(15,22,23,27,32,35)$. Although the prognostic value of the $K R A S$ mutation is still controversial, several studies suggest a poor prognosis $(24,35,36)$. Since $N R A S$ is a $R A S$ family member, we expected $N R A S$-mutated CRC patients to have a poor prognosis compared with those without $R A S$ mutations. However, multivariate analysis showed a tendency toward a better prognosis for $N R A S$-mutated CRC patients. Therefore, the present study suggests that CRC cases with NRAS mutations exhibit different characteristics than CRC cases with KRAS mutations.

Recent studies report that common KRAS exon 2 mutations targeting the RAS/RAF/MAPK pathway are currently used to determine patient eligibility for anti-EGFR monoclonal antibody therapy $(19,37,38)$. Minor mutations (KRAS exon 3 and 4, NRAS, BRAF) are expected to become the next predictive biomarkers. In fact, a recent clinical trial on anti-EGFR monoclonal antibodies excluded patients with the KRAS exon 2 mutation and those with these less frequent mutations (39). In the present study, the incidence of $K R A S$ exon 2 mutations was $38 \%$, whereas the combined mutation rate for $K R A S$ exon 3 and $4, N R A S$ and $B R A F$ was $49.3 \%$. For stage IV CRC patients subjected to anti-EGFR monoclonal antibody therapy, the total mutation rate increased from 37.3 to $51.6 \%$. If the validity of excluding CRC patients with these minor $R A S$ mutations from anti-EGFR monoclonal antibody therapy is verified by future trials, more than $50 \%$ of CRC patients would greatly benefit from personalized medicine for enhanced efficacy and a better prognosis.

In conclusion, the present study demonstrated that $K R A S$ - and NRAS-mutated CRC tumors exhibit distinct characteristics and distributions along the colorectum. Future molecular biology studies should address the significance of these differences between NRAS- and KRAS-mutated CRC and confirm possible positive prognoses associated with NRAS mutations. 


\section{Acknowledgements}

We would like to thank Akemi Takahashi, Mina Yamada and Syuhei Takahashi for their excellent technical assistance. The present study was supported by the Japanese Ministry of Health, Labour and Welfare.

\section{References}

1. Scaltriti $\mathrm{M}$ and Baselga $\mathrm{J}$ : The epidermal growth factor receptor pathway: a model for targeted therapy. Clin Cancer Res 12: 5268-5272, 2006.

2. Mendelsohn $\mathrm{J}$ and Baselga J: Epidermal growth factor receptor targeting in cancer. Semin Oncol 33: 369-385, 2006.

3. Fang JY and Richardson BC: The MAPK signalling pathways and colorectal cancer. Lancet Oncol 6: 322-327, 2005.

4. Schubbert S, Shannon K and Bollag G: Hyperactive Ras in developmental disorders and cancer. Nat Rev Cancer 7: 295-308, 2007.

5. Ginesta MM, Mora J, Mayor R, et al: Genetic and epigenetic markers in the evaluation of pancreatic masses. J Clin Pathol 66 : 192-197, 2013.

6. Mascaux C, Iannino N, Martin B, et al: The role of RAS oncogene in survival of patients with lung cancer: a systematic review of the literature with meta-analysis. Br J Cancer 92: 131-139, 2005.

7. Kohlmann A, Grossmann V, Klein HU, et al: Next-generation sequencing technology reveals a characteristic pattern of molecular mutations in $72.8 \%$ of chronic myelomonocytic leukemia by detecting frequent alterations in TET2, CBL, RAS, and RUNX1. J Clin Oncol 28: 3858-3865, 2010.

8. Uhara $\mathrm{H}$, Ashida A, Koga $\mathrm{H}$, et al: NRAS mutations in primary and metastatic melanomas of Japanese patients. Int J Clin Oncol: June 6, 2013 (Epub ahead of print).

9. De Roock W, Claes B, Bernasconi D, et al: Effects of $K R A S$, $B R A F, N R A S$, and PIK3CA mutations on the efficacy of cetuximab plus chemotherapy in chemotherapy-refractory metastatic colorectal cancer: a retrospective consortium analysis. Lancet Oncol 11: 753-762, 2010.

10. Vaughn CP, Zobell SD, Furtado LV, Baker CL and Samowitz WS Frequency of $K R A S, B R A F$, and NRAS mutations in colorectal cancer. Genes Chromosomes Cancer 50: 307-312, 2011.

11. Loupakis F, Ruzzo A, Cremolini C, et al: KRAS codon 61, 146 and $B R A F$ mutations predict resistance to cetuximab plus irinotecan in $K R A S$ codon 12 and 13 wild-type metastatic colorectal cancer. Br J Cancer 101: 715-721, 2009.

12. Irahara N, Baba Y, Nosho K, et al: NRAS mutations are rare in colorectal cancer. Diagn Mol Pathol 19: 157-163, 2010.

13. Seymour MT, Brown SR, Middleton G, et al: Panitumumab and irinotecan versus irinotecan alone for patients with $K R A S$ wild-type, fluorouracil-resistant advanced colorectal cancer (PICCOLO): a prospectively stratified randomised trial. Lancet Oncol 14: 749-759, 2013.

14. Smith CG, Fisher D, Claes B, et al: Somatic profiling of the epidermal growth factor receptor pathway in tumors from patients with advanced colorectal cancer treated with chemotherapy \pm cetuximab. Clin Cancer Res 19: 4104-4113, 2013.

15. Farina-Sarasqueta A, van Lijnschoten G, Moerland E, et al: The BRAF V600E mutation is an independent prognostic factor for survival in stage II and stage III colon cancer patients. Ann Oncol 21: 2396-2402, 2010.

16. Van Cutsem E, Kohne CH, Hitre E, et al: Cetuximab and chemotherapy as initial treatment for metastatic colorectal cancer. N Engl J Med 360: 1408-1417, 2009.

17. Bokemeyer C, Bondarenko I, Makhson A, et al: Fluorouracil, leucovorin, and oxaliplatin with and without cetuximab in the first-line treatment of metastatic colorectal cancer. J Clin Oncol 27: 663-671, 2009.

18. Karapetis CS, Khambata-Ford S, Jonker DJ, et al: K-ras mutations and benefit from cetuximab in advanced colorectal cancer. N Engl J Med 359: 1757-1765, 2008.

19. Douillard JY, Siena S, Cassidy J, et al: Randomized, phase III trial of panitumumab with infusional fluorouracil, leucovorin, and oxaliplatin (FOLFOX4) versus FOLFOX4 alone as firstline treatment in patients with previously untreated metastatic colorectal cancer: the PRIME study. J Clin Oncol 28: 4697-4705, 2010 .
20. Amado RG, Wolf M, Peeters M, et al: Wild-type $K R A S$ is required for panitumumab efficacy in patients with metastatic colorectal cancer. J Clin Oncol 26: 1626-1634, 2008.

21. Douillard JY, Oliner KS, Siena S, et al: Panitumumab-FOLFOX4 treatment and $R A S$ mutations in colorectal cancer. N Engl J Med 369: 1023-1034, 2013

22. Samowitz WS, Sweeney C, Herrick J, et al: Poor survival associated with the $B R A F$ V600E mutation in microsatellite-stable colon cancers. Cancer Res 65: 6063-6069, 2005.

23. Roth AD, Tejpar S, Delorenzi M, et al: Prognostic role of KRAS and $B R A F$ in stage II and III resected colon cancer: results of the translational study on the PETACC-3, EORTC 40993, SAKK 60-00 trial. J Clin Oncol 28: 466-474, 2010.

24. Imamura Y, Morikawa T, Liao X, et al: Specific mutations in $K R A S$ codons 12 and 13 , and patient prognosis in $1075 B R A F$ wild-type colorectal cancers. Clin Cancer Res 18: 4753-4763, 2012.

25. Lochhead P, Kuchiba A, Imamura Y, et al: Microsatellite instability and $B R A F$ mutation testing in colorectal cancer prognostication. J Natl Cancer Inst 105: 1151-1156, 2013.

26. Gavin PG, Colangelo LH, Fumagalli D, et al: Mutation profiling and microsatellite instability in stage II and III colon cancer: an assessment of their prognostic and oxaliplatin predictive value. Clin Cancer Res 18: 6531-6541, 2012.

27. Ogino S, Shima K, Meyerhardt JA, et al: Predictive and prognostic roles of $B R A F$ mutation in stage III colon cancer: results from intergroup trial CALGB 89803. Clin Cancer Res 18: $890-900,2012$.

28. Akagi K, Uchibori R, Yamaguchi K, Kurosawa K, Tanaka Y and Kozu T: Characterization of a novel oncogenic K-ras mutation in colon cancer. Biochem Biophys Res Commun 352: 728-732, 2007.

29. Asaka S, Arai Y, Nishimura Y, et al: Microsatellite instabilitylow colorectal cancer acquires a KRAS mutation during the progression from Dukes' A to Dukes' B. Carcinogenesis 30: 494-499, 2009

30. Ishikubo T, Nishimura Y, Yamaguchi K, et al: The clinical features of rectal cancers with high-frequency microsatellite instability (MSI-H) in Japanese males. Cancer Lett 216: 55-62, 2004.

31. Andreyev HJ, Norman AR, Cunningham D, Oates JR and Clarke PA: Kirsten ras mutations in patients with colorectal cancer: the multicenter 'RASCAL' study. J Natl Cancer Inst 90: 675-684, 1998.

32. Yokota T, Ura T, Shibata N, et al: BRAF mutation is a powerful prognostic factor in advanced and recurrent colorectal cancer. $\mathrm{Br}$ J Cancer 104: 856-862, 2011.

33. Oh JR, Kim DW, Lee HS, et al: Microsatellite instability testing in Korean patients with colorectal cancer. Fam Cancer 11: 459-466, 2012.

34. Yamauchi M, Morikawa T, Kuchiba A, et al: Assessment of colorectal cancer molecular features along bowel subsites challenges the conception of distinct dichotomy of proximal versus distal colorectum. Gut 61: 847-854, 2012.

35. Richman SD, Seymour MT, Chambers P, et al: KRAS and BRAF mutations in advanced colorectal cancer are associated with poor prognosis but do not preclude benefit from oxaliplatin or irinotecan: results from the MRC FOCUS trial. J Clin Oncol 27: 5931-5937, 2009.

36. Phipps AI, Buchanan DD, Makar KW, et al: KRAS-mutation status in relation to colorectal cancer survival: the joint impact of correlated tumour markers. Br J Cancer 108: 1757-1764, 2013.

37. Tol J, Koopman M, Cats A, et al: Chemotherapy, bevacizumab, and cetuximab in metastatic colorectal cancer. N Engl J Med 360: 563-572, 2009 .

38. Bokemeyer C, Bondarenko I, Hartmann JT, et al: Efficacy according to biomarker status of cetuximab plus FOLFOX-4 as first-line treatment for metastatic colorectal cancer: the OPUS study. Ann Oncol 22: 1535-1546, 2011.

39. Fornaro L, Lonardi S, Masi G, et al: FOLFOXIRI in combination with panitumumab as first-line treatment in quadruple wild-type (KRAS, NRAS, HRAS, BRAF) metastatic colorectal cancer patients: a phase II trial by the Gruppo Oncologico Nord Ovest (GONO). Ann Oncol 24: 2062-2067, 2013. 